Communication

\title{
Designing a Novel Nano-Vaccine against SARS-CoV-2
}

\author{
Ang Gao ${ }^{1,2 \#}$, Hui Liang ${ }^{2 \#}$, Qi Shen², Cheng Zhou², Xiao Min Chen², Jing Tian², \\ Xueling $\mathrm{Li}^{2}$, Zexi Liu', Jian $\mathrm{Ni}^{1}$, Daxiang Cui ${ }^{1,2}$ \\ ${ }^{1}$ Institute of Nano Biomedicine and Engineering, Shanghai Engineering Research Centre for Intelligent Diagnosis and Treatment \\ Instrument, Department of Instrument Science and Engineering, School of Electronic Information and Electrical Engineering, \\ Shanghai Jiao Tong University, 800 Dong Chuan RD, Shanghai 200240, PR China. \\ ${ }^{2}$ National Engineering Research Center for Nanotechnology, 28 East Jiang Chuan Road,Shanghai 200241, China. \\ \# These authors contributed equally to this work. \\ C Corresponding authors. E-mail: dxcui@sjtu.edu.cn; 13818096617@139.com
}

Received: Aug. 24, 2020; Accepted: Oct. 27, 2020; Published: Oct. 28, 2020

Citation: Ang Gao, Hui Liang, Qi Shen, Cheng Zhou, Xiao Min Chen, Jing Tian, Xueling Li, Zexi Liu, Jian Ni, and Daxiang Cui, Designing a Novel Nano-Vaccine against SARS-CoV-2. Nano Biomed. Eng., 2020, I2(4): 32 I-324.

DOI: 10.5101/nbe.v12i4.p321-324.

\begin{abstract}
The new coronavirus SARS-CoV-2 has become a global pandemic, which has had a huge impact on the lives of people around the world and has caused huge impacts and losses on global economic development. To now, there is still no effective drug or therapy against coronavirus. A large number of studies have shown that vaccines are the ultimate weapon to eliminate major infectious diseases. The development of new vaccines against new coronaviruses is the best way to prevent new coronavirus infections. In this study, we developed a new vaccine against the new coronavirus by combining our self-developed nano adjuvant loaded with carnosine graphene oxide adjuvant with loaded with CpG molecule and RBD protein antigen. Our results showed that this vaccine can produce high titer antiSARS-CoV-2 RBD antibody neutralizing SARS-CoV-2 in mice within 2 weeks.
\end{abstract}

\section{Keywords: COVID-1, Vaccine, Neutralizing antibodie}

Up to now, more than 20 million people across the globe have been diagnosed with COVID-19, with nearly 750,000 deaths, and showing a rising trend in global. Most of significant, Chloroquine and Remdesivir have been used as antiviral drugs in clinical trials for the treatment of COVID-19, The final results both show that the effects of these drugs are limited [1, 2].

At present, only immunosuppressive drugs, like dexamethasone, have been proved to have an exact effect in relieving COVID-19 Severe symptoms [3]. Additionally, a series of serious sequela has been found in recovered patients. The clinical studies have found find neutralize antibody for SARS-CoV-2 in the plasma from recovered patients. This indicated that vaccines may be an effective method for COVID-19. A vaccine for severe acute respiratory syndrome coronavirus 2 (SARS-CoV-2) is needed to control the global coronavirus infectious disease (COVID-19) public health crisis.

Since the outbreak of the SARS CoV-2 epidemic, more than 170 vaccines have been developed, and 26 of them have entered into a clinical study, of which 7 have entered the phase III clinical stage. And these vaccines could be divided into inactivated virus vaccine, genetic vaccine (DNA \& RNA), virus vector vaccine, recombination protein vaccine. Among these vaccine types, the inactivated virus vaccine 
can trigger the immune response and establish an immune defense system against the COVID-19 by injecting the inactivated virus. This approach has a long development cycle, and inactivated vaccines of Novartis and Beijing Institute of Biological Products have entered phase III clinical trials [4]; Nucleic acid vaccines can deliver encoding DNA or RNA of the COVID-19 into normal cells and translate specific viral proteins, thus induce the recognition of viral proteins by the immune system. This method is simple and fast, and can be designed quickly after the viral nucleic acid sequence is sequencing, but so far there is no DNA/ RNA vaccine has no drug been approved, still need more clinical to prove its efficacy and safety [5, 6]. Moderna and Pfizer mRNA vaccines have successively entered phase III clinical trials. Viral vector vaccine is to transfect the gene of the COVID-19 into the harmless virus. These recombinant viruses infect humans and achieve immunity to the COVID-19. However, this method still relies on the infection of humans by viral vectors. Once a part of the people has been immunized against the viral vectors, these recombinant viruses' vaccine could lose its efficiency. The Oxford adenovirus vaccine will complete Phase III clinical trials in September 2020, and the adenovirus vaccine developed by CanSino has entered Phase III clinical trials to further evaluate its safety and efficacy $[7,8]$. The last one is the recombinant protein vaccine, which synthesizes the COVID-19 specific antigen in vitro by bacteria or cell lines. After vaccination with adjuvant, leukocytes could quickly respond and recognize of the vaccine, then produce neutralizing antibodies [9]. Recombinant protein vaccine has the advantages of high safety and response sensitivity. Novavax recombinant protein vaccine has entered phase III clinical trials, and the vaccine developed by the Chinese Academy of Sciences has also entered phase II clinical trials [10]. In conclusion, the rapid response and saturated $R \& D$ strategy with a variety of new technologies and new theories make the speed of vaccine research and development increased dramatically. It is expected that the vaccine for COVID-19 will be on the market at the end of 2020 . Comparing with mRNA vaccine, the recombinant $S$ protein vaccine has predominant stability and efficiency, and the foundation of a biopharmaceutical can strongly support the complex manufacturing process. For the threaten of the COVID-19 pandemic in the next winter, a recombinant $S$ protein vaccine may be more suitable.
Toll-like receptor 9 (TLR9) is expressed in a variety of B-cell malignancies and works as a bridge between innate and adaptive immunity. CpG oligodeoxynucleotides (CpG ODNs), TLR9 agonists, can induce immune responses and exert direct effects against disease, serving as vaccine adjuvant or monotherapy. CpG has been widely used in clinical studies [11]. To improve the immune activity of immunogens and enhance the immune response-ability, the most basic method is to mix immunogens with adjuvants, which can enhance the immune response of the body to immunogens. Many literature and experimental data show that $\mathrm{CpG}$ oligodeoxynucleotide (ODN) has the effect of activating the body's immune system, and CpG ODN is a quite promising adjuvant discovered in recent years [12]. How to achieve systemic targeting of $\mathrm{CpG}-\mathrm{ODN}$ to a specific immune cell is a problem, that needs we have to solve.

As a conventional inorganic nanomaterial, graphene has excellent drug loading ability due to its unique $\pi-\pi$ conjugation at every single graphene layer, which can efficiently adsorb small molecular and macromolecular drugs, and provides an excellent delivery platform for nucleic acid and protein delivery (Fig. 1) [13]. However, its stable two-dimensional structure and chemical properties also greatly limit the release rate of drugs in vivo, these characteristics make conventional drugs unable to reach the therapeutic window and play a therapeutic effect. These shortcomings seem fatal, but fit our needs for the COVID-19 vaccine design.



Fig. 1 The Scheme of GOCR Vaccine structure.

In this study, we developed a convenient and efficient COVID-19 vaccine GOCR Vaccine. The vaccine preparation can be accomplished simply by mixing $\mathrm{CpG}$ and the spike RBD protein of SARSCoV-2 with graphene oxide carriers' solution. After the vaccine was inoculated subcutaneously in mice, RBD could achieve slow and long-term control release, uninterruptedly stimulate the immune system, and produce a high titer neutralizing antibody to prevent the invasion of COVID-19. Because of the 

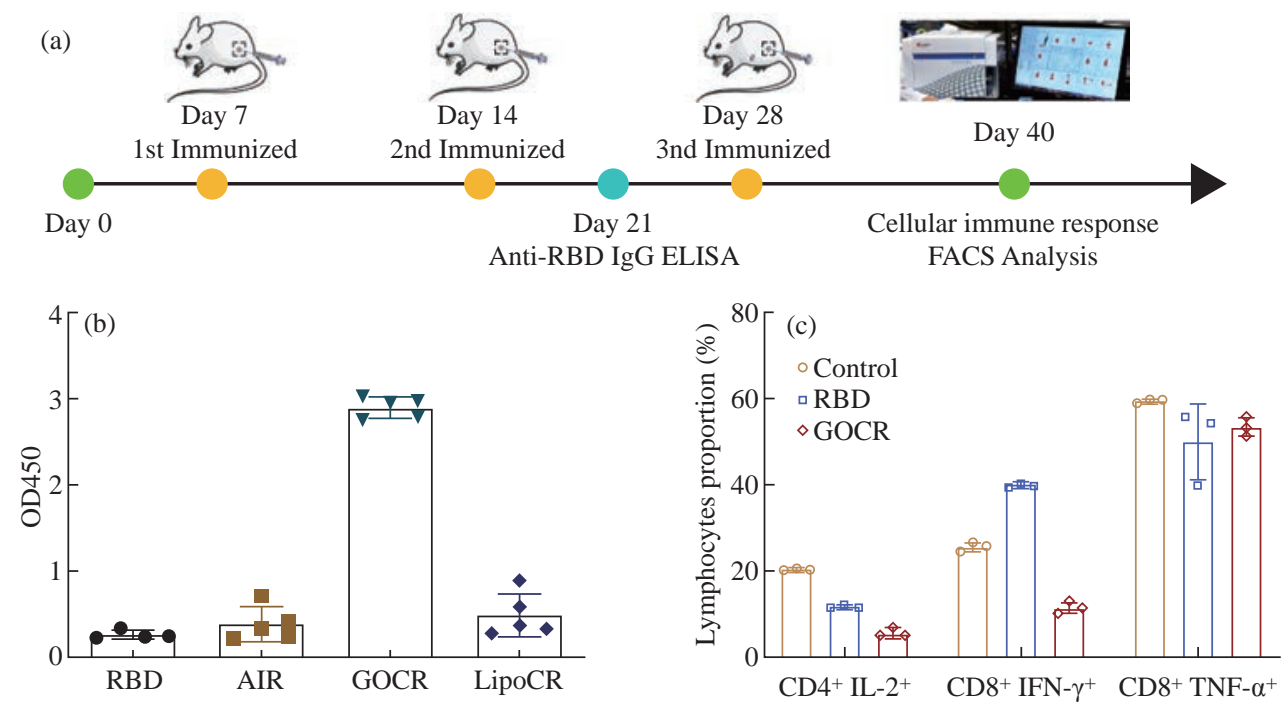

Fig. 2 The production of anti-SARS-CoV-2 RBD neutralizing antibody and analysis of cellular immunity. (a) Route of GOCR vaccine administration. (b) The production of anti-SARS-CoV-2 RBD neutralizing antibody. (c) FACS analysis of cytokine in spleen lymphocytes after vaccination.

simple preparation process and low cost of the GOCR Vaccine, it is very suitable for large-scale production. This technology also provides a simple and effective vector for the development of the COVID-19 vaccine and other recombinant protein vaccines.

After that, we analyzed neutralizing antibodies in immunized mice after two vaccinations. ELISA assay of neutralizing antibody production in mouse serum showed that the graphene-based vaccine GOCR Vaccine could induce a large number of neutralizing RBD antibodies in vivo (Fig. 2). Compared with the traditional aluminum adjuvant, the neutralizing antibody production increased at least 7 times. This result strongly demonstrated that the GOCR Vaccine had a great advantage over the traditional vaccine and could strongly stimulate the immune system, and produce higher titer antibodies to combat the invasion of COVID-19. Next, we evaluated the effect of graphene-based vaccines on cellular immunity. By flow cytometric analysis of splenic lymphocytes in mice, we found that the vaccine would not promote the secretion of proinflammatory cytokines in large quantities, thus did not cause strong cellular immunity and did not trigger a cytokine storm. At the same time, there were no significant weight differences among the groups following vaccination, this proved the safety of this vaccine.

In summary, we have designed an efficient vaccine and confirmed the production of neutralizing antibodies after vaccination. We will continue to study the neutralizing activity of RBD antibodies derived from plasma to pseudoviruses and live viruses. Furthermore, we will further explore the degradation of graphene in vivo and hope to construct a safety and biocompatibility vaccine.

\section{Acknowledgements}

This work was supported by Key Basic Research Program of China (No. 2017YFA0205304), Nature Scientific Foundation of China (No. 81602184), and Medical Engineering Cross Project of Shanghai Jiao Tong University (YG2017ZD12). This work was also supported by "the Belt and Road" young scientist exchange program of the Science and Technology Commission of Shanghai (Grant No. 18410741600).

\section{Conflict of Interests}

The authors declare no conflict of interest.

\section{References}

[1] M. Mahévas, V.T. Tran, M. Roumier, et al., Clinical efficacy of hydroxychloroquine in patients with covid-19 pneumonia who require oxygen: observational comparative study using routine care data. Bmj, 2020, 369: $\mathrm{m} 1844$.

[2] C.D. Spinner, R.L. Gottlieb, G.J. Criner, et al., Effect of remdesivir vs standard care on clinical status at 11 days in patients with moderate COVID-19: A randomized clinical trial. Jama, 2020.

[3] P. Horby, W.S. Lim, J.R. Emberson, et al., Dexamethasone in hospitalized patients with Covid-19-preliminary report. N Engl J Med, 2020. 
[4] H. Wang, Y. Zhang, B. Huang, et al., Development of an inactivated vaccine candidate, BBIBP-CorV, with potent protection against SARS-CoV-2. Cell, 2020, 182: 713721. e9.

[5] T.R.F. Smith, A. Patel, S. Ramos, et al., Immunogenicity of a DNA vaccine candidate for COVID-19. Nat Commun, 2020, 11: 2601.

[6] D. Yin, S. Ling, X. Tian, et al., A single dose SARSCoV-2 simulating particle vaccine induces potent neutralizing activities. BioRxiv, 2020: 093054.

[7] F.C. Zhu, Y.H. Li, X.H. Guan, et al., Safety, tolerability, and immunogenicity of a recombinant adenovirus type5 vectored COVID-19 vaccine: a dose-escalation, openlabel, non-randomised, first-in-human trial. Lancet, 2020, 395: $1845-1854$.

[8] N. van Doremalen, T. Lambe, A. Spencer, et al., ChAdOx1 nCoV-19 vaccine prevents SARS-CoV-2 pneumonia in rhesus macaques. Nature, 2020.

[9] P.J. Hotez, M.E. Bottazzi, Developing a low-cost and accessible COVID-19 vaccine for global health. PLoS Negl Trop Dis, 2020, 14: e0008548.

[10] L. Dai, T. Zheng, K. Xu, et al., A universal design of betacoronavirus vaccines against COVID-19, MERS, and SARS. Cell, 2020, 182: 722-733.

[11] M.A. Anwar, M. Shah, J. Kim, et al., Recent clinical trends in Toll-like receptor targeting therapeutics. Med Res Rev, 2019, 39: 1053-1090.

[12] M. Liu, R.S. O’Connor, S. Trefely, et al., Metabolic rewiring of macrophages by $\mathrm{CpG}$ potentiates clearance of cancer cells and overcomes tumor-expressed CD47mediated 'don't-eat-me' signal. Nat Immunol, 2019, 20: 265-275.

[13] C. Meng, X. Zhi, C. Li, et al., Graphene oxides decorated with carnosine as an adjuvant to modulate innate immune and improve adaptive immunity in vivo. ACS Nano, 2016, 10: 2203-2213.

Copyright $₫$ Ang Gao, Hui Liang, Qi Shen, Cheng Zhou, Xiao Min Chen, Jing Tian, Xueling Li, Zexi Liu, Jian Ni, and Daxiang Cui. This is an open-access article distributed under the terms of the Creative Commons Attribution License, which permits unrestricted use, distribution, and reproduction in any medium, provided the original author and source are credited. 